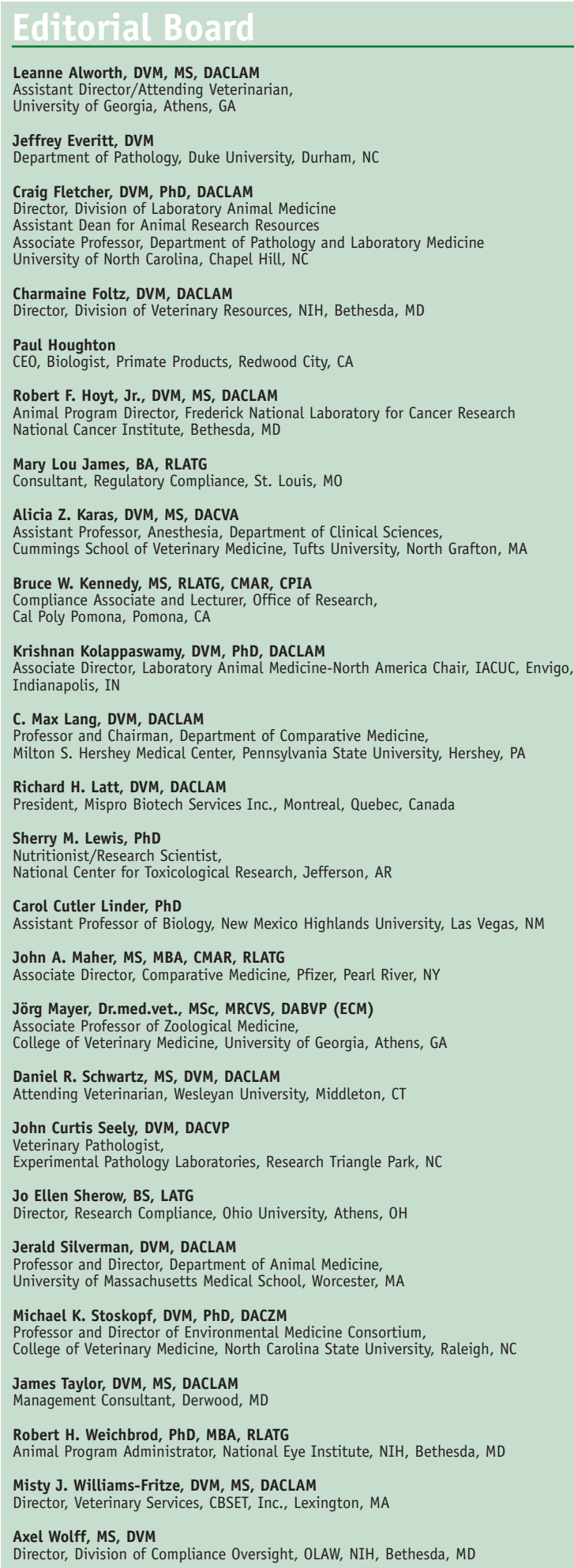

\section{Stop the fighting}

Though a normal behavior, aggression between mice is a considerable concern in the laboratory as fighting can lead to serious injury and death or euthanasia. Unlike in the wild, laboratory mice in their home cages have limited means of avoiding or de-escalating aggressive interactions with their cage mates. Vivarium staff and researchers have devised numerous ways to keep the peace, but with limited empirical evidence. In a new research article this month, Brianna Gaskill, Kathleen Pritchett-Corning and their colleagues present the results of their investigation into several developmental, environmental, and genetic factors thought to influence home-cage aggression. They analyze pelt aggression lesion scores to test whether weaning age, group composition, scent treatment, substrain, environmental enrichment, or identification methods influence aggression-related wounding in recently shipped male C57BL/6 mice.

See page 176

\section{A wider spectrum of phenotypes and alternatives}

Central nervous system disorders can manifest very differently in humans than in animals; our attempts to model complex neurodegenerative phenotypes in animals like mice in order to find potential treatments have had limited translational success. Correspondence from Allan Kalueff offers suggestions to improve CNS disease research and drug development. One way is to expand the spectrum and our understanding of accepted phenotypes for a given disease, and to encourage community-driven efforts to share novel endpoints. Another is to expand the list of accepted model species to include increasingly important alternatives, like zebrafish.

See page 91

\section{Clarifying new terms}

A USDA bulletin released last fall has introduced two new terms to inspection reports and the Animal Care Inspection Service (ACIS) database: focused inspections and critical noncompliant items. To address some community concerns, Policy Watch explains these terms and provides examples from the database of how they have turned up-if under a different name-in past reports. The changes should prove to be useful, making it easier for those interested to find relevant information from the ACIS database going forward. See page 85 